\section{Effect of Lunar Eclipse on the Ionosphere}

For some time we have been studying the variation of height of the different layers of the ionosphere at different hours of the day and night by the well-known group-retardation method. The technique of Breit and Tuve', with the later improvements suggested by Appleton and Builder ${ }^{2}$, has been adopted for emitting short radio pulses from an aerial system of half-wave Hertzian horizontal dipole type, fed by a Lecher system. The receiver with the recording system of cathode ray oscillograph is located at a distance of about $1.5 \mathrm{~km}$. from the transmitter. It may be mentioned that the average equivalent height of the Kennelly-Heaviside, or $E$ layer, is found to be $100 \mathrm{~km}$., and that of the Appleton, or $F$ layer, about $270 \mathrm{~km}$. The observations have been taken with waves having frequency of $3 \cdot 8$ megacycles per second.

During the lunar eclipse of January 8, 1936, a whole-night observation showed that the echoes from the $F$ layer, which were prominent before the eclipse, became substantially feeble during the totality between the hours 2330 and 2354 I.S.T. The reflected waves regained their former strength after the eclipse. This observation indicates that the moon has also some contributory effect in changing the ionisation of the upper atmosphere. The ioncontent becomes appreciably minimised during the absence of the moon. This presumably may be due to the presence of ultra-violet waves which appear in the lunar spectrum ${ }^{3}$.

\section{S. S. BANERJEE.} B. N. SINGH.

Physics Laboratory,

Benares Hindu University, Benares, India. Jan. 27.

${ }^{1}$ Breit and Tuve, Phys. Rev., 28, 554 (1926)

2 Appleton and Builder, NATrRE, 127,970 (June 27, 1931).

${ }^{3}$ Hanson and Hulburt, Phys. Rev., 37, 477 (1931).

\title{
Points from Foregoing Letters
}

THE results of research on the carbon dioxide content of air under various conditions, carried out immediately prior to his death by Prof. J. S. Haldane, together with Dr. R. H. Makgill, are outlined by Prof. J. B. S. Haldane. The influence of height above ground, vegetation, seasons, soil, sea, city surroundings, etc., is indicated. The simplicity and rapidity of the method devised may render it useful in other fields, for example, for distinguishing, in meteorological observations, between bodies of air of different origin.

Dr. Albert Fischer finds that by transferring a few drops of a solution $(A)$ of serum globulin (at $70^{\circ} \mathrm{C}$.), in process of coagulation, to a fresh solution $(B)$ of the same substance, the rate of induced coagulation in $(B)$ depends upon the rate in $(A)$ at the moment of transfer. From the retarding effect of alkali, he infers that such chain reactions, leading to the denaturation of proteins, may be explained by the appearance of radicals $\left(-\mathrm{NH}_{2},-\mathrm{SH}\right)$ on the surface of the spherical protein molecules.

Diagrams showing the atomic arrangements in crystalline powders of prussian blue and related pigments, as deduced from X-ray investigations, are submitted by J. F. Keggin and F. D. Miles.

Absorption of light by several of the com. pounds of hæmoglobin (red pigment of the blood) has been investigated by G. A. Adams. These substances show a common absorption point in the region of $4100 \mathrm{~A}$., which is apparently due to the porphyrin ring system in 'hæm' (a basic compound the hydrochloride of which is hæmin, a bluish-black decomposition product of hæmoglobin).

By using Geiger-Müller counters (for counting ionising particles) with walls made of nickel and of tin, and irradiating them with slow neutrons, $R$. Naidu finds that induced radioactive substances are produced. In the case of nickel, the half-life is three hours, while in the case of tin two half-periods of 8 and 18 minutes respectively are observed.

The secondary emission of molybdenum with various amounts of barium surface contamination has been studied by L. R. G. Treloar. A definite relation has been established between secondary emission and work function, provided that the contamination is not more than one atom thick.

A new possible way in which electrons can escape from the cathode of an are is indicated by Dr. M. J. Druyvesteyn; if the cathode is covered with a very thin layer of an insulating substance, the number of positive ions on its surface can produce a high field which will enable electrons to escape from the metal. and penetrate through this insulator into the gas with a high velocity, without recombining with the ions on the surface.

According to Drs. I. Ramakrishna Rao and C. Sambasiva Rao, a study of the Raman effect in strong electrolytes suggests that, while all molecules with electrovalent bonds are completely dissociated even in highly concentrated solutions, those with covalent bonds (possible only between the anion and the hydrogen ion) change progressively with dilution into the electrovalent type and thus towards com. plete dissociation.

John F. Marshall and J. Staley report observations on the mosquito Theobaldia subochrea which indicate that this species possesses the rare characteristics of mating within small cages and laying fertile eggs with. out a previous meal of blood.

A case of transference of the mite, Tarsonemus pallidus, from cyclamen to a strawberry plant is reported by D. O. Boyd and W. E. H. Hodson.

The existence in the fat-soluble extract of kale or alfalfa of a new vitamin the absence of which leads to gizzard erosion in chicks, is indicated by experiments reported by $H$. J. Almquist and E. L. R. Stokstad. The authors show that the needed dietary factor is distinct from that responsible for the prevention of hæmorrhage in chicks.

Y. D. Wad and F. K. Jackson of the Institute of Plant Industry, Indore, show that humic composts and inorganic fertilisers should be regarded as complementary to each other and not necessarily competing. For the most efficient use of inorganic nutrients, soil organic matter must be in proper condition and must reach a certain level. If the necessary amount, which varies with the crop, is not present, humic composts may be employed to adjust the level. 